\title{
Torsional Splittings and Assignments of the Doppler-Limited Spectrum of Ethane in the C.H Stretching Region*
}

\author{
A. S. Pine** and W. J. Lafferty** \\ National Bureau of Standards, Washington, DC 20234
}

November 18, 1981

\begin{abstract}
The Doppler-limited absorption spectrum of the C-H stretching region of ethane has been recorded at $T \simeq 119 \mathrm{~K}$ with a tunable difference-frequency laser spectrometer. The strong torsional hot band structure at room temperature is eliminated at $119 \mathrm{~K}$, and the enhanced resolution from the Doppler width reduction allows us to observe small torsional splittings. The two fundamentals in the region, $v_{7}$, a perpendicular band and, $v_{5}$, a parallel band have been essentially completely assigned as have a large number of transitions in the parallel component of the $v_{8}+v_{11}$ combination band. A number of perturbations of both global and local nature have been observed. The complete spectrum and a listing of transition wavenumbers, intensities and assignments are presented here to facilitate identification and quantitative analysis of ethane in a variety of monitoring applications. Precise ground state rotational constants have been determined from combination differences.
\end{abstract}

Key words: C-H stretching region; difference- frequency laser; Doppler-limited resolution; ethane; ground state constants; infrared spectrum; low temperature spectrum; torsional splittings.

\section{Introduction}

Although ethane is the simplest hydrocarbon containing a saturated carbon-carbon bond and has very high symmetry $\left(D_{3 d}\right)$, the extremely dense and complex rotational fine structure of its infrared bands has defied complete resolution until recent advances in Doppler-limited tunable laser and Fourier transform instrumentation. Previously, high-quality grating spectra of the C-H stretching region at $0.025 \mathrm{~cm}^{-1}$ resolution permitted Cole, Lafferty and Thibault $[1]^{1}$ to partially assign the rotational transitions of the $v_{7}$ perpendicular band. More recently Cole, Cross, Cugley and Heise [2] deconvolved similar grating data to $\sim 0.015 \mathrm{~cm}^{-1}$ to observe somewhat more structure in this same band. We present here the Doppler-limited absorption spectrum of the infrared active $\mathrm{C}-\mathrm{H}$ stretches of ethane recorded at $T \simeq$ $119 \mathrm{~K}$ (Doppler FWHM $=0.0043 \mathrm{~cm}^{-1}$ ) with a tunable difference-frequency laser spectrometer. Both fundamentals, $v_{7}$ centered at $2985.39 \mathrm{~cm}^{-1}$ and the parallel band $v_{5}$ at $2895.67 \mathrm{~cm}^{-1}$, have been fully resolv-

*The experimental portion of this work was performed while the author was with Lincoln Laboratory of the Massachusetts Institute of Technology under NSF Contract \#NSF/ASRA/DAR 78-24562. A preliminary account of this work appears in the NSF Final Report entitled "Tunable Laser Survey of Molecular Air Pollutants: Doppler-Limited Spectra of the C-H Stretching Bands of Formaldehyde, Ethylene, Ethane and Allene," January 1980.

**Molecular Spectroscopy Division, National Measurement Laboratory.

${ }^{1}$ Figures in brackets indicate literature references at the end of this paper. ed and assigned, and much of the $v_{8}+v_{11}$ combination band has been identified.

The principal complications in the infrared spectrum of $\mathrm{C}_{2} \mathrm{H}_{6}$ arise from the relative torsional motion of the two methyl groups about the saturated C-C bond [3]. The moderate potential barrier (of $\sim 1024 \mathrm{~cm}^{-1}$ ) to free internal rotation leads to a low frequency torsional mode $v_{4}$ at $\sim 290 \mathrm{~cm}^{-1}$ as determined by a number of calorimetric [4,5] and spectroscopic [6,7] techniques. This mode is highly excited at room temperature giving rise to "hot bands" associated with each normal band originating in the ground vibrational state. These hot bands are effectively suppressed in the present study by maintaining the ethane sample at the lowest possible temperature above the vapor condensation point. Overtones and combination states of this low frequency torsional mode are also in Fermi or Coriolis resonance with virtually all the higher vibrations of the molecule, causing severe perturbations whose complexity increases dramatically with the wavenumber of the other bands because of the increasing number of possible combinations.

Tunneling through the torsional potential barrier creates a further doubling of the energy levels of the molecules which may be manifest as small splittings in the rovibrational transitions as discussed by Susskind [8]. However, for nontorsional modes, such as those studied here, the barrier and, hence, the torsional split- 
tings are not expected to vary significantly from the ground state. Thus the splittings for nontorsional infrared transitions, which arise from the difference in the splittings of the upper and lower vibrational levels [8], are expected to be unresolvable, even at the Doppler limit. Nevertheless the resonant interactions with torsional overtones and combinations, mentioned above, induce variations in the barrier giving rise to observable torsional splittings in the present work. Some of these are illustrated in figure 1 for the region around ${ }^{R} Q_{5}$ of the $v_{7}$ band, as brought out by the resolution enhancement and spectrum simplification resulting from lowering the ethane temperature. The torsional doublets of the $R_{R_{2}}(7)$ and $R_{R_{3}}(4)$ lines and the interleaved ${ }^{R} Q_{5}$ subbranch are unambiguously characterized by their nuclear spin statistical weight intensity ratios calculated by Wilson [9].

The first observation of torsional splitting in ethane was made in diode laser spectra of the $v_{9}$ band by Patterson, Flicker, McDowell and Nereson [10]. These splittings also arise from perturbations, induced by a resonance with $3 v_{4}$; but since that is the only possible

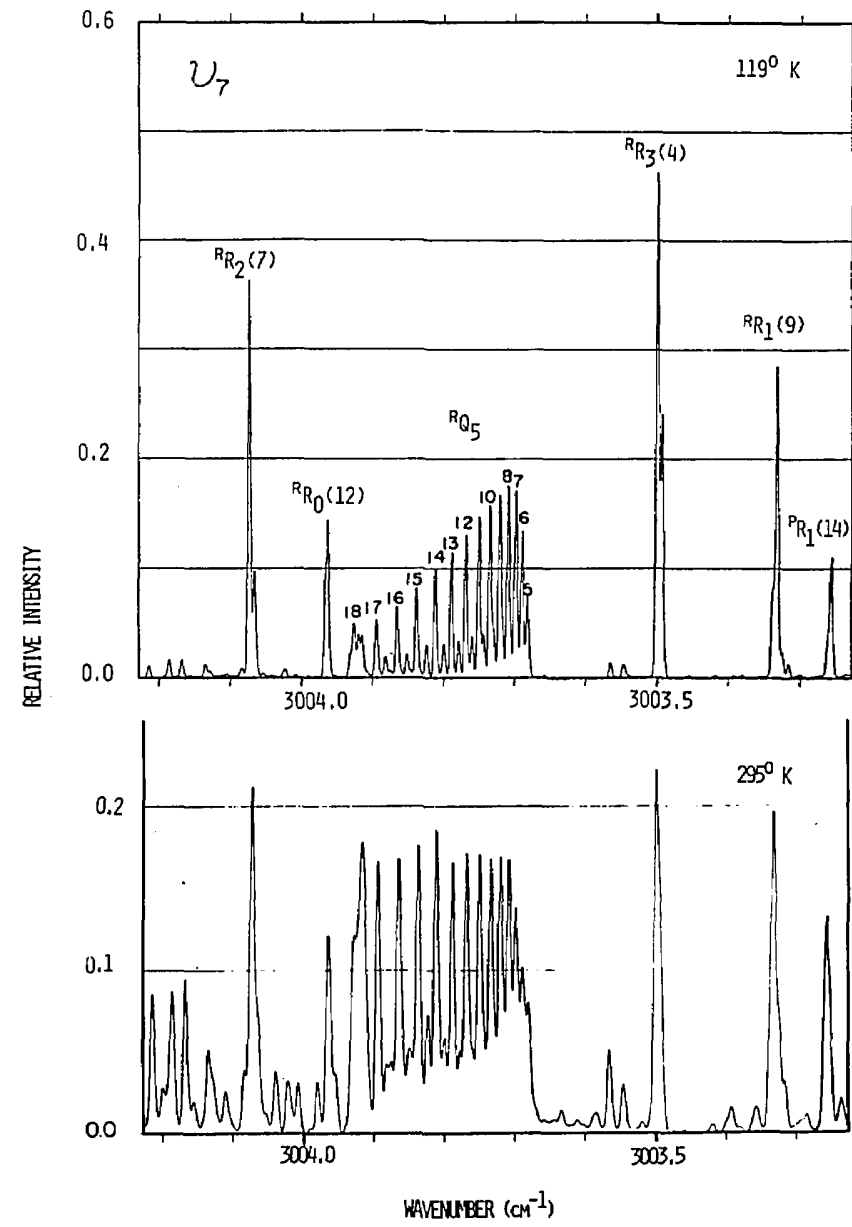

FIGURE 1. Comparison of the ethane spectrum in the region of the ${ }^{R} Q_{5}$ subbranch of the $v_{7}$ band for temperatures of $119 \mathrm{~K}$ and $295 \mathrm{~K}$. resonance with $\nu_{9}$, that band is more amenable to analysis than the complex C-H stretching bands. Two detailed studies of the $\nu_{9}$ band are now in progress $[11,12]$; both are based on Doppler-limited diode laser spectra of the $Q$ branches and comprehensive, precision Fourier transform interferometer data. In the present study of the C-H stretches, the torsional splittings exhibit many interesting patterns indicative of a variety of interactions and perturbing states.

The complex low temperature Doppler-limited spectrum of ethane between 3051 and $2862 \mathrm{~cm}^{-1}$ is given in this report along with a listing of transition wavenumbers, intensities and assignments. We also discuss the assignment procedure and the more interesting spectral features associated with the perturbations, torsional splittings and combination bands. A precise set of ground state rotational constants obtained from combination differences is given since microwave data do not exist for this nonpolar molecule. These definitive spectra are truly characteristic of the molecule without instrumental distortion since the laser linewidth $\left(\sim 0.0003 \mathrm{~cm}^{-1}\right)$ is much narrower than the Doppler width. Hence, the spectral patterns should be useful for the identification and quantitative analysis of ethane for a number of practical applications. For example, ethane is a principal constituent of natural gas 10 to $20 \%$ with $\sim 7 \%$ average) and can be used for labelling sources, detecting leaks and monitoring pipeline transmission and LNG carriers [13]; it is one of the major hydrocarbon emissions in automobile exhausts and can interfere with the measurement of more toxic components [14]; and it has been observed in the atmosphere of several outer planets [15] with significance to extraterrestrial organic chemistry.

\section{Experimental Considerations}

The high resolution ethane spectra reported here were recorded with a tunable difference-frequency laser spectrometer developed at MIT Lincoln Laboratory for obtaining precise and comprehensive Doppler-limited vapor-phase spectra in the 2.2 to $4.2 \mu \mathrm{m}$ region. The instrument is based on the nonlinear optical downconversion of $\mathrm{CW}$ visible single-mode argon ion and tunable dye lasers in a $\mathrm{LiNbO}_{3}$ mixing crystal. The basic operating characteristics of the system [16] along with techniques of drift compensation [17], stabilization and automated data processing [18] and linear scan control [19] have been detailed elsewhere, so we will discuss here only those features pertinent to the present investigation.

Since the C-H stretching bands of ethane are quite extensive (covering $\sim 200 \mathrm{~cm}^{-1}$ ), the difference-frequency system was operated in a broad survey, rapid scan mode. The absorption spectra were recorded in continuously 
scanned $3.75 \mathrm{~cm}^{-1}$ segments overlapped at intervals of 3 $\mathrm{cm}^{-1}$. The scan rate was $0.0125 \mathrm{~cm}^{-1} / \mathrm{s}$ or $375 \mathrm{MHz} / \mathrm{s}$ so each segment was scanned in 5 minutes. The postdetection time constant was $40 \mathrm{~ms}$ (corresponding to $5 \mathrm{x}$ $10^{-4}$ or $15 \mathrm{MHz}$ ) which was short enough to allow full response to the sharp Doppler-broadened lines (FWHM $=128 \mathrm{MHz}$ for $\mathrm{C}_{2} \mathrm{H}_{6}$ at $T \simeq 119 \mathrm{~K}$ ) but long enough for good signal-to-noise ratio of $\sim 500$. The transmission traces were digitized at a $20 \mathrm{~Hz}$ rate $(6000$ points per segment, $6.25 \times 10^{-4} \mathrm{~cm}^{-1}$ per point) for data storage and subsequent numerical processing. The instrumental width, due primarily to the dye laser free-running jitter, was about $10 \mathrm{MHz}$ FWHM. Thus the effect of instrumental broadening is negligible since convolution of the Gaussian profiles of the Doppler and laser distributions results in the widths adding in quadrature.

The ethane spectra were recorded over a period of 2 days. Each day's run was calibrated against $\mathbf{C H}_{4}$ reference lines from the Fourier transform interferometer data of Tarrago et al. [20]. The first day's run was interpolated between the $R(3)$ and $P(7)$ manifolds of the $v_{3}$ band of $\mathrm{CH}_{4}$ while that of the second day between $P(7)$ and $P(15)$ using a $5 \mathrm{~cm}$ confocal Fabry-Perot interferometer with a free spectral range of $0.050027(1)$ $\mathrm{cm}^{-1}$. The interorder spacing of this scan calibration interferometer can be measured to a precision of $\pm 2 \times 10^{-7}$ $\mathrm{cm}^{-1}$ over a $100 \mathrm{~cm}^{-1}$ interval, but it exhibits a small frequency dispersion and overnight thermal shifts which must be checked during extended runs. The determination of the spectal line wavenumbers for these rapid survey scans was made to a relative precision of $\sim \pm 4 \mathrm{x}$ $10^{-4} \mathrm{~cm}^{-1}$ which was slightly better than the least reading for the digitizing grid. The absolute accuracy of the wavenumbers may be slightly worse due to uncertainties in the reference line standards.

In order to obtain linestrength information from the transmission traces, one must account for Beer's law, $\alpha(\omega)=(\varrho L)^{-1} \ln (B(\omega) / S(\omega))$. Here $\alpha(\omega)$ is the absorption coefficient in units of $(\varrho L)^{-1}$ where $\varrho$ is the sample density and $L$ is the cell length; $S(\omega)$ is the transmission and $B(\omega)$ is the empty cell baseline. In this case the baseline was entered manually on a coarse grid $\left(0.125 \mathrm{~cm}^{-1}\right)$ since it was not practical to evacuate the cold cell and refill for each trace. Also our automatic baseline interpolation routine, which keys on the background between lines, did not work reliably for ethane since the spectrum was so dense. The manual baseline estimation is only adequate to $\sim 1$ percent because of strong channeling of the spectra from the cold cell windows. The baseline uncertainties dominate the relative line strength errors since the actual transmission spectra are reproducible to $\simeq \pm 0.2$ percent. In addition, absolute intensities depend on measurement accuracy of the cell length $(29.4 \pm 0.1$ cm), the fill pressure $(225 \pm 4 \mathrm{~m}$ Torr at $T=295 \pm 1$
$\mathrm{K}$ ), the sample temperature (held at $T=119 \pm 3 \mathrm{~K}$ ) and, to a lesser extent, on sample purity. Here we used natural isotopic ethane of research grade from Matheson with a quoted purity of 99.96 percent; it exhibited no trace of methane or other simple hydrocarbons in the spectrum. In the atlas, corrections are made for the isotopic abundance of ${ }^{12} \mathrm{C}_{2} \mathrm{H}_{6}$.

The sample cold cell was a copper tube with thinned end sections for stress-free epoxy mounting of $\mathrm{ZnSe}$ windows required because of differential thermal contraction. Copper cooling coils were soldered to the tube and an evacuable stainless steel jacket with $\mathrm{CaF}_{2}$ windows provided thermal isolation. The cell was cooled by manually controlling the flow of $\mathrm{He}$ gas through a liquid $\mathrm{N}_{2}$ heat exchanger and then through the cell coils. The temperature was measured with a single platinum resistance thermometer imbedded in the copper cell wall. The uniformity of the temperature is expected to be better than the manually controlled temperature setting which dominates the absolute intensity errors $(\sim \pm 3 \%$ for low $J$ and $K$ ).

\section{Spectral Features}

The fundamentals, $\nu_{5}$ and $v_{7}$, are examples of parallel and perpendicular bands whose assignment, as is customary, is based on selection rules, "missing lines" and consistent ground state combination differences. Where observed, the torsional doublets provide a definitive confirmation of the $K$ and some $J$ assignments because of their distinctive intensity ratios for particular rotational quantum numbers. The nuclear spin statistical weights for these doublets derived by Wilson [9] yield the intensity ratios of $4: 1$ for $K$ not a multiple of 3 ; $2: 1$ for $K$ a multiple of 3 but not 0 ; and for $K=0$ a ratio of 3:1 for $J$ even and 5:3 for $J$ odd. These ratios are illustrated in figure 1 for $K=2,3$ and 5 levels of $\nu_{7}$ and in figure 2 for the $K=0,2$ and 4 cases in the $P(6)$ and $P(7)$ manifolds of $\nu_{5}$. Each torsional component is labelled in the atlas according to the ground state symmetry species of the permutation-inversion group $G_{36}^{\dagger}$ for ethane-like molecules exhibiting internal rotation as discussed by Hougen [21].

Though the assignments for the fundamentals are firm and rather complete, a number of severe perturbations are observed due to accidental resonant crossings of energy levels. However, for both $\nu_{5}$ and $v_{7}$ the initial $K$ subband lines at $J=K$ appear regular and undisturbed. This implies that both bands are affected by X-Y Coriolis interactions since the matrix elements governing such a resonance are proportional to $[J(J+1)-K(K+$ $1)]^{1 / 2}$. In most cases the effect of such a perturbation is to change the effective $B$ values of individual subbands but to leave the $K$ structure unaffected. However, for the 


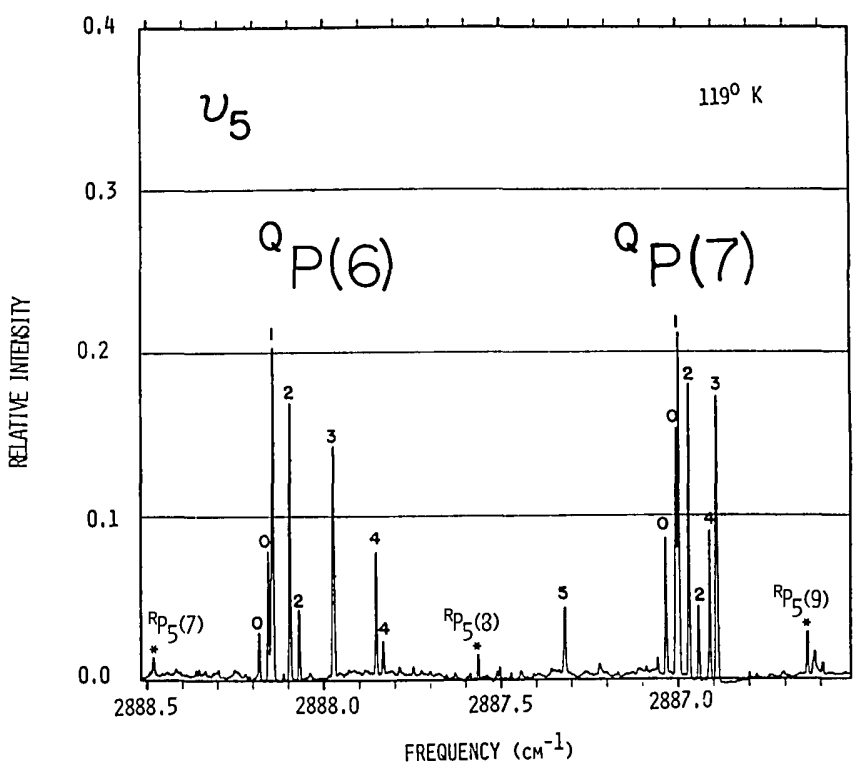

Figure 2. Spectrum of $\mathrm{C}_{2} \mathrm{H}_{6}$ near the $P(6)$ and $P(7)$ manifolds of the $\nu_{5}$ band at $T=119 \mathrm{~K}$. Perturbation-induced lines are labelled with an*.

very close resonances observed in some of the subbands here, the perturbations are severe enough that they cannot be fit using a standard power series expansion. In $v_{7}$ such a resonance is dramatically exhibited by the ${ }^{R} Q_{5}$ subbranch shown in figure 1 . This subbranch is much more spread out than lower ${ }^{R} Q_{K}$ subbranches and is degraded in opposite direction to the higher ${ }^{R} Q_{K}$ subbranches; it also shows an abrupt head and gap above $J$ $=18$. In addition, there are numerous smaller local perturbations, one of which, shown in figure 3 , occurs in the

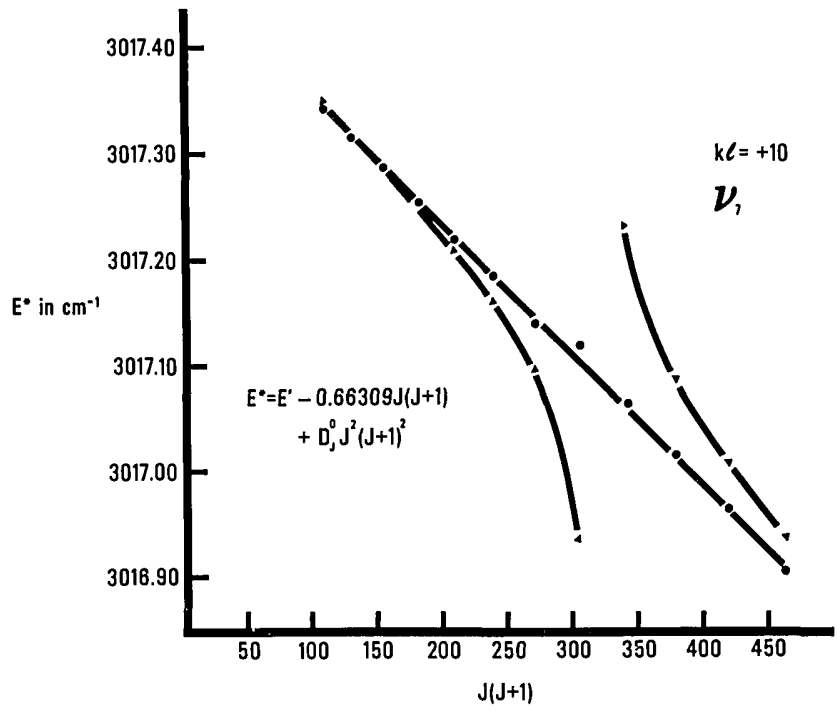

Figure3. Torsional splittings in the $K^{\prime}=10, \Delta K=+1$ subband of $v_{7}$. The dots represent the stronger $A_{1 s}-A_{2 s}$ symmetry components and the triangles represent the weaker $E_{3 s}-E_{48}$ symmetry components.
$K=10, \Delta K=+1$ subband, manifest in a rapid increase in the torsional splitting as a function of $J$ to a value $\sim 0.1 \mathrm{~cm}^{-1}$ at $J^{\prime}=17$, above which it suddenly reverses sign and decreases again to higher $J^{\prime}$. In some subbands of $v_{7}$ the torsional splitting is constant or a monotonic function of $J(J+1)$ and in others it appears to be irregular.

On the other hand, perturbations in $\nu_{5}$ appear to be dominated by a strong resonance near the $K=5$ energy level stack. The effect of this resonance is to degrade the $K=5$ subband to lower frequency while the others are degraded to higher frequency as shown in figure 4. Despite the degradation, the subband "origins" at $J=$ $K$ fall on a relatively unperturbed straight line, as seen in figure 4, implicating the X-Y Coriolis interaction. From

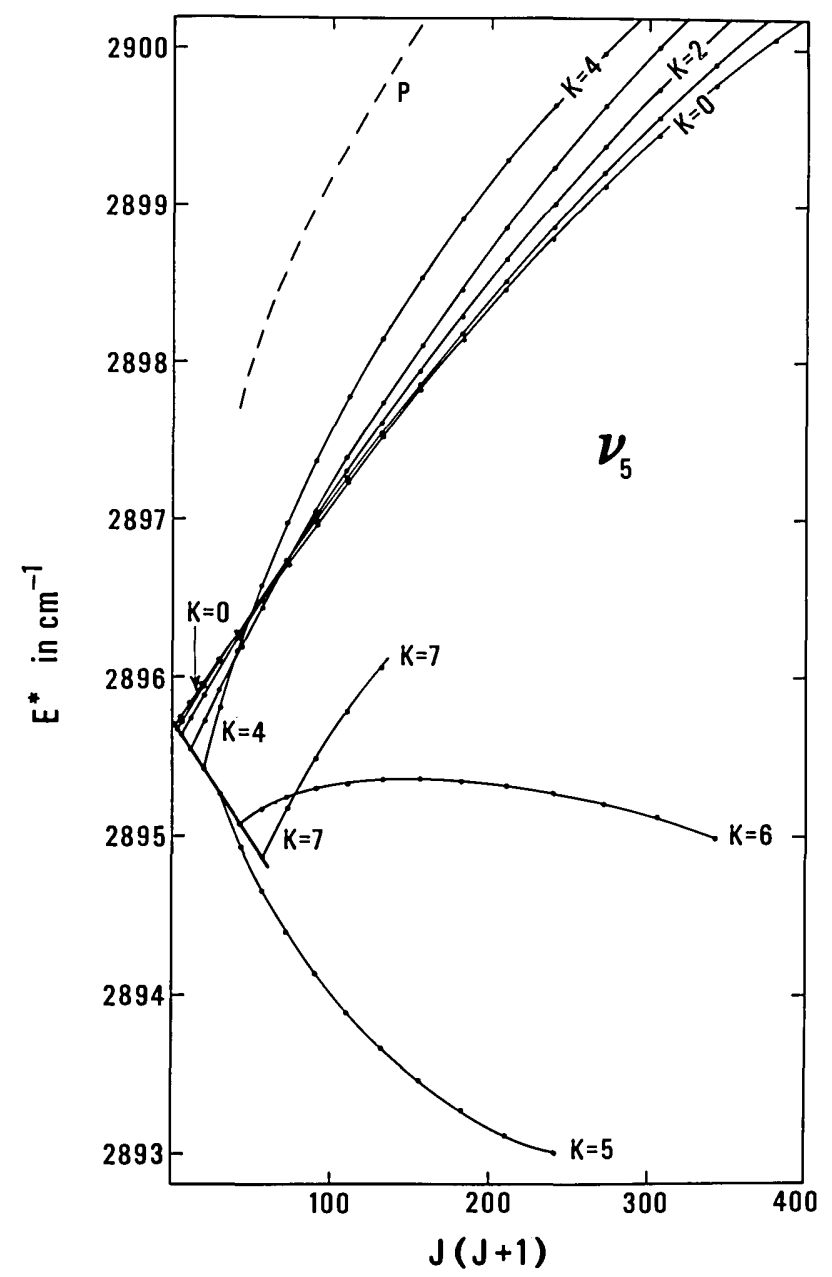

Figure 4. Reduced energy for the stronger torsional components of the $K$ subbands of $v_{5}$. The dashed line represents the $K=6$ subband of an $E_{\mathfrak{u}}$ vibration perturbing $K=5$ of $v_{5}$ and observed by resonant mixing. Here $E^{*}=v_{5}\left(J^{\prime} K, J^{\prime \prime} K^{\prime \prime}\right)+B_{\sigma}^{\prime \prime \prime}\left(j^{\prime \prime}+1\right)-B_{5}^{*} J^{\prime}\left(J^{\prime}\right.$ $+1)$ where $v_{5}\left(J^{\prime} K^{\prime}, J^{\prime \prime} K^{\prime \prime}\right)$ are the transition wavenumbers for the band and $B_{5}^{*}=0.6624 \mathrm{~cm}^{-1}$ is chosen to adjust the scale of the plot. 
the $a_{2 u}$ symmetry of $\nu_{5}$ and the $E_{\mathrm{g}}$ species for the vibrational operator of the X-Y Coriolis coupling, we can conclude that the perturbing vibration has $E_{u}$ symmetry. The resonance mixing is strong enough near the crossover that the perturbing levels accompanying the $K$ $=4$ and 5 subbands borrow sufficient intensity to be observed in the spectrum. The levels perturbing $K=5$ are positioned along the dashed curve, labelled $P$ in figure 4, and a few of the corresponding perturbationinduced transitions are shown in figure 2, labelled with an asterisk. We believe that these perturbing levels belong to the $K=6$ subband of the resonant state because of the $\Delta K= \pm 1$ requirement for the $X-Y$ Coriolis interaction and the fact that the $J=K=5$ level of $\nu_{5}$ is unperturbed. The $K$ assignments for the perturbating subbands must be regarded as tentative since we have so few series, but they are consistent with the intensity ratios of their torsional doublets which have splittings comparable to those in $\nu_{5}$.

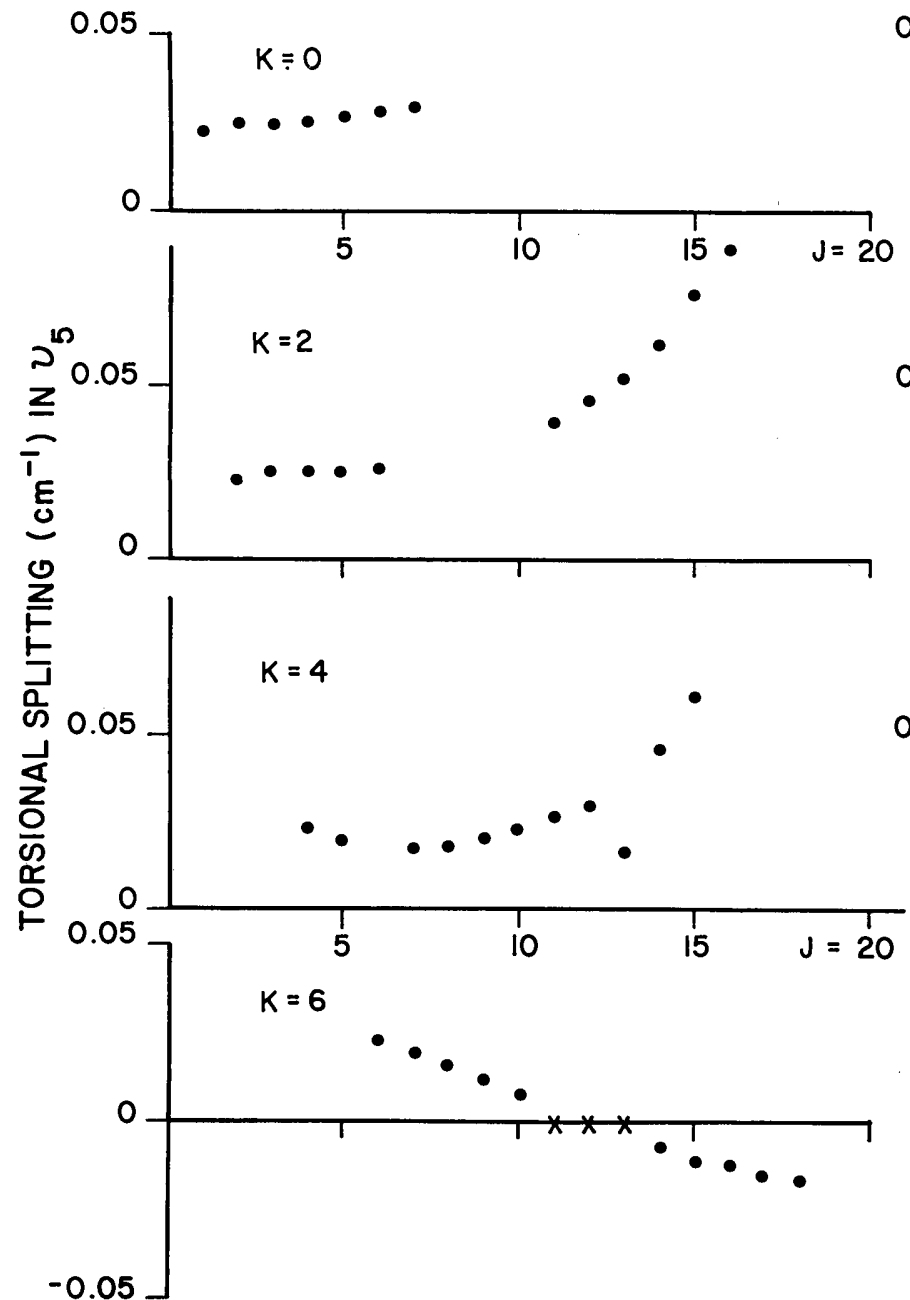

The perturbation-induced torsional splittings take the interesting form shown in figure 5 . For the odd $K$ subbands the $J=K$ transitions are not observably split, but at higher $J$ the splitting increases regularly, to as much as $\sim 0.1 \mathrm{~cm}^{-1}$ in some cases. Even for the most perturbed subband, $K=5$, the doublet of the $J=5$ transition is not resolved while $J=6$ is. In the even $K$ subbands however, the $J=K$ transitions are all split by $\sim 0.02$ $\mathrm{cm}^{-1}$ and, except for $K=6$, the splittings increase slowly with $J$. For $K=6$ the splittings decrease with $J$, become unresolved at about $J=14$ and are resolved again at higher $J$ with the splittings reversed. We currently have no consistent model for this striking behavior of the torsional splittings in $\nu_{5}$. It is clear however, that recognizing the presence of torsional doubling was the key to assigning $v_{5}$ which was missing in prior studies $[1,2]$.

The combination bands present a somewhat more difficult situation to analyze. First there are ambiguities in
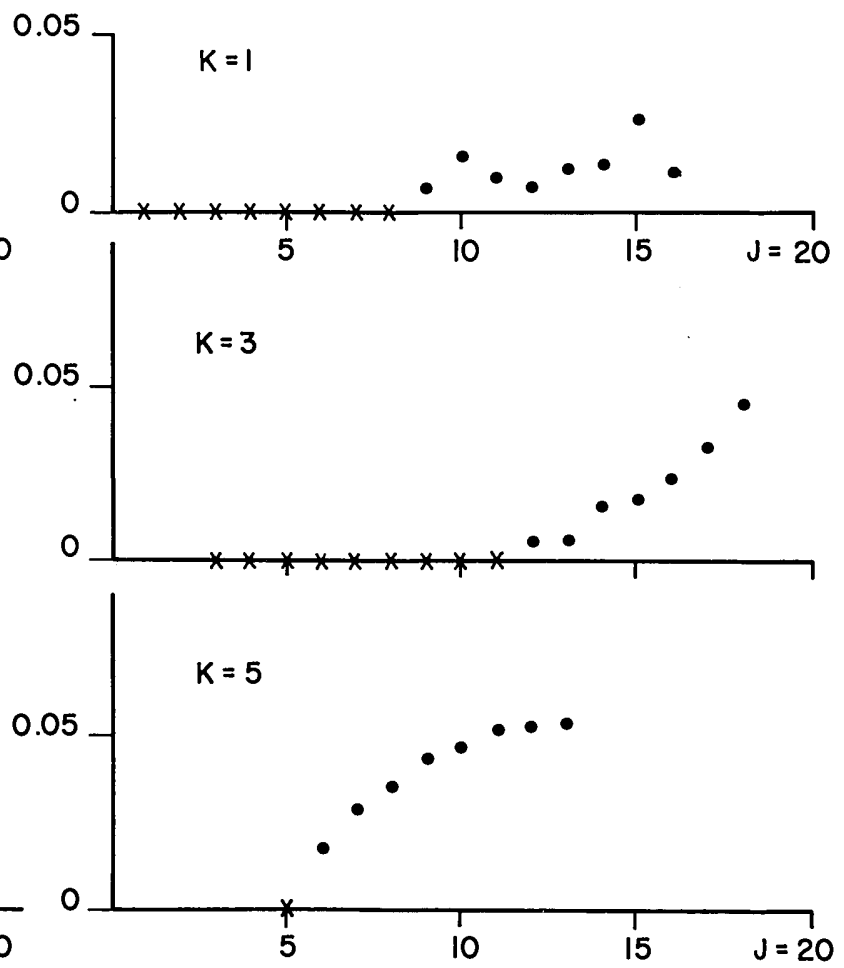

Figure 5. Torsional splittings in the $v_{5}$ band of $\mathrm{C}_{2} \mathrm{H}_{6}$ for $0 \leqslant K \leqslant 6$. Here $\mathrm{X}$ represents unresolved doublets. 
the vibrational sublevel symmetries for the $v_{8}+v_{11}$ combination band since there are several components possible $\left(e_{u} \times e_{g}=A_{1 u}+A_{2 u}+E_{u}\right)$. The $E_{u}$ component of $v_{8}+v_{11}$ should result in a perpendicular band with an effective $\xi_{+}=-\left(\xi_{8}+\xi_{11}\right) \simeq+0.71$ from the data of Nakagawa and Shimanouchi [22], leading to ${ }^{P}, R_{Q_{K}}$ subbranches spaced by $\sim 7.8 \mathrm{~cm}^{-1}$. We see no direct evidence for such a band in our spectrum. On the other hand, we see at least two moderately strong parallel bands belonging to the $A_{u}$ components. To first order only the $A_{2 u}$ mode should be infrared active, but the normally inactive $A_{1 u}$ mode may borrow intensity from $A_{2 u}$ via a Z-type Coriolis interaction proportional to $H_{12}=-A K \xi_{-}$, where $\xi_{-}=I \xi_{8}-\xi_{11} \mathrm{I}$, as discussed by Hougen [23]. The appearance of the $A_{1}-A_{2}$ band also depends on the separation, $\Delta E$, between these two states. In the uncoupled limit where either $\Delta E$ is large or $\mathrm{H}_{12}$ is small, the mixing is negligible and only transitions to the ordinary $A_{2 u}$ parallel band would be observed. If $\Delta E=$ 0 the mixing will be complete and the resulting infrared band will have the appearance of a perpendicular band (with parallel band intensity patterns [24]) with a Coriolis constant $\xi_{-}$. Intermediate cases require a more detailed calculation. The two modes can be distinguished by the presence of a $K=0$ subband allowed for $A_{2 u}$ and forbidden for $A_{1 u}$ since the Coriolis coupling is proportional to $K$.

A number of parallel-type subbands were assigned in this region by first identifying strong series in the $R$ branch with estimated $J$ and $K$ and calculating the $Q$ and $P$ lines using the precise ground state combination differences obtained from the fundamental bands. If the series matches observed lines to within $\sim 0.0005 \mathrm{~cm}^{-1}$ and the intensities are consistent, then one can be quite confident of the $J$ assignment. The $K$ assignment is less certain because the combination differences are not strongly $K$ dependent. However again, where torsional splittings are observed ( $K=0$ and 3 in this instance) the $K$ assignments are verified by the doublet intensity ratios. The other subbands were assigned $K$ values according to the missing lines resulting from $J \geqslant K$. In the $P$ and $R$ branches, the lower $J$ transitions are weak and often obscured and overlapped by stronger series. For the $Q$ branch of a parallel band, the lowest $J$ line is strongest, so the $Q$ branches were most useful in establishing the $K$ numbering of the series. One subband has a very small value of $B^{\prime}-B^{\prime \prime}$ and its $Q$ subbranch, falling at $2958.0863 \mathrm{~cm}^{-1}$, is not resolved. This subband is tentatively believed to involve $K=2$ transitions with an uncertainty of \pm 1 in $K$. The prominent feature in the spectrum at about $2953.8 \mathrm{~cm}^{-1}$ consists of $Q$ subbranches ranging from $K=2$ to 6 . The lower $K$ subbands have origins both above and below this feature; namely $K=0$ at $2955.6699(2) \mathrm{cm}^{-1}, K=1$ at
$2956.1196(4) \mathrm{cm}^{-1}$ and $2951.9962(2) \mathrm{cm}^{-1}$ and $K=2$ at $2958.0866(8) \mathrm{cm}^{-1}$.

Although the majority of lines occuring in the combination band region have been assigned, their subbands are badly perturbed and many strong series remain unidentified. The $v_{8}$ and $v_{11}$ band is expected to have both Fermi and Coriolis interactions with the fundamentals and with other higher order overtones and combinations. Also Hougen [25] has pointed out the possibility of an interaction between the $A_{1 u}$ component and the totally symmetric $a_{1 g}$ C-H stretching mode, $v_{1}$, in ethane-like molecules with an intermediate barrier. This interaction, strictly forbidden for rigid $D_{3 d}$ molecules, is permitted during the torsional tunneling motion. It is of course expected to be weak in ethane but the near degeneracy with the Raman-active $v_{1}$ mode at 2953.7 $\mathrm{cm}^{-1}$ as determined by Lepard, Shaw and Welsh [26] may account for some of the observed anomalies in this band.

\section{Ground State Constants}

Since ethane is nonpolar, no microwave data are available for the ground state rotational constants. Thus we have calculated the rotational constants from the large number of ground state combination differences (CDs) collected during the course of the assignment of the C-H stretching bands. We have restricted the CDs used in obtaining the constants to those obtained from the two fundamentals, $\nu_{5}$ and $\nu_{7}$, since the lines from these bands are stronger, more precisely measured than those in the combination band, and the assignment is firm in these bands. In calculating CDs from transitions which were split into torsional components, the lines were assigned the ground state symmetry of Hougen [21] according to the observed intensity ratios, and differences were taken between components of the same symmetry. A number of lines were unsplit and, if these lines were sharp, CDs between them were also included in the fit. Lines for which the torsional splitting was only poorly resolved were not included in the fit.

The ground state constants of a prolate symmetric top molecule are defined by the usual term value equation:

$$
\begin{aligned}
F(J, K)= & \left(A_{\mathrm{o}}-B_{\mathrm{o}}\right) K^{2}+B_{\mathrm{o}} J(J+1) \\
& -D_{\mathrm{o}}^{J} J^{2}(J+1)^{2}-D_{\mathrm{o}}^{\mathrm{JK}} K^{2}(J+1)-D_{\mathrm{o}}^{K} K^{4} .
\end{aligned}
$$

No "forbidden" transitions with | $\Delta K$ | $>1$ were observed in the spectrum so only the coefficients of the $J$ dependent terms can be determined. A total of $766 \mathrm{CDs}$ were obtained from the fundamental bands of which two thirds were from torsionally split lines. Originally CDs of each symmetry species were fit separately to determine if there were any dependence of the rotational constants on 
symmetry or the corresponding nuclear spin. No statistically significant differences were found so all CDs were then fit simultaneously, resulting in the ground state constants given in table 1.

TABLE 1: Ground State Constants of ${ }^{12} \mathrm{C}_{2} \mathrm{H}_{6}$ in $\mathrm{cm}^{-1}$

$\begin{array}{ll}A_{0} & =2.671^{(\mathrm{a})} \\ B_{0} & =0.6630271(14)^{(\mathrm{b})} \\ D_{0}^{K} & =1.09 \times 10^{-5}(\mathrm{c}) \\ D_{0}^{J K} & =2.660(29) \times 10^{-6(b)} \\ D_{0}^{J} & =1.0312(26) \times 10^{-6(b)}\end{array}$

"Raman value for Ref. [26].

bPresent work with uncertainties in parentheses representing one standard deviation.

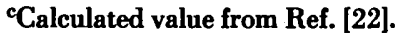

Not only does the combination difference fitting provide an excellent set of rotational constants, but it also reflects the measurement precision obtained in this experiment. The standard deviation of the fit is $\mathbf{0 . 0 0 0 5 5}$ $\mathrm{cm}^{-1}$ or $16.5 \mathrm{MHz}$. Since the ground state fitting is performed on differences, the measurement uncertainty of an individual line is reduced by a factor of $1 / \sqrt{2}$ to $\sim 12$ MHz. These ground state constants, given with near microwave precision, are certainly an improvement over prior lower resolution grating spectra determinations, and they are compatible with ultra precise Fourier transform interferometer data on $v_{9}$ currently being analyzed [11].

\section{Spectrum and Listing}

We present in figure $6^{2}$ the complete Doppler-limited absorption spectrum of ethane from $3051 \mathrm{~cm}^{-1}$ to 2870 $\mathrm{cm}^{-1}$ recorded at $T \simeq 119 \mathrm{~K}$. Prominent $\mathrm{Q}$ branches are labelled for $\nu_{7}$, and the $K=0$ subband lines for the $P(J)$ and $R(J)$ manifolds are labelled for $\nu_{5}$. A listing of the measured transitions is given in table 2 consisting of the line center wavenumber, the peak intensity, the upper and lower state rotational quantum numbers when assigned, the ground state vibration-rotationaltorsion symmetry in the $G_{36}^{\dagger}$ group [21] when resolved (the subscript $s$ is suppressed), the vibrational band code and the lower state energy. The vibrational band code is $A$ for $v_{7}, B$ for $v_{8}+v_{11}, C$ for $v_{5}$, and $D$ for perturbation-induced lines. The lower state energy in wavenumbers is calculated from eq (1) using the precise rotational constants of table 1 and the more approximate values for $A_{\mathrm{o}}$ and $D_{\mathrm{o}}^{K}$ obtained from Raman spectra [26]. These ground state functions [3], are needed to compute the temperature dependence of the line intensities.

The intensity scale for figure 6 is given in (Torr $m)^{-1}$ taken from the "raw" experimental fill pressure at room temperature and the cell length. However, the experiment was conducted at constant density since the cell was sealed when the temperature was lowered. Thus for the listing of table 2 we convert the peak intensity units to (Amagat-cm) $)^{-1}$ by multiplying (Torr $\left.-\mathrm{m}\right)^{-1}$ by the factor $(760 / 100) \times(295 / 273) / a=8.397$ where $a=$ 0.978 is the isotopic abundance of ${ }^{12} \mathrm{C}_{2} \mathrm{H}_{6}$.

The authors are grateful to A. Mooradian and P. M. Moulton of MIT Lincoln Laboratory for the loan of the cold cell. They are also indebted to J. T. Hougen for numerous helpful discussions on the torsional symmetry and interactions in ethane.

\footnotetext{
${ }^{2}$ Figure 6 and table 2 are displayed on pages 244-256. References are on page 256.
} 

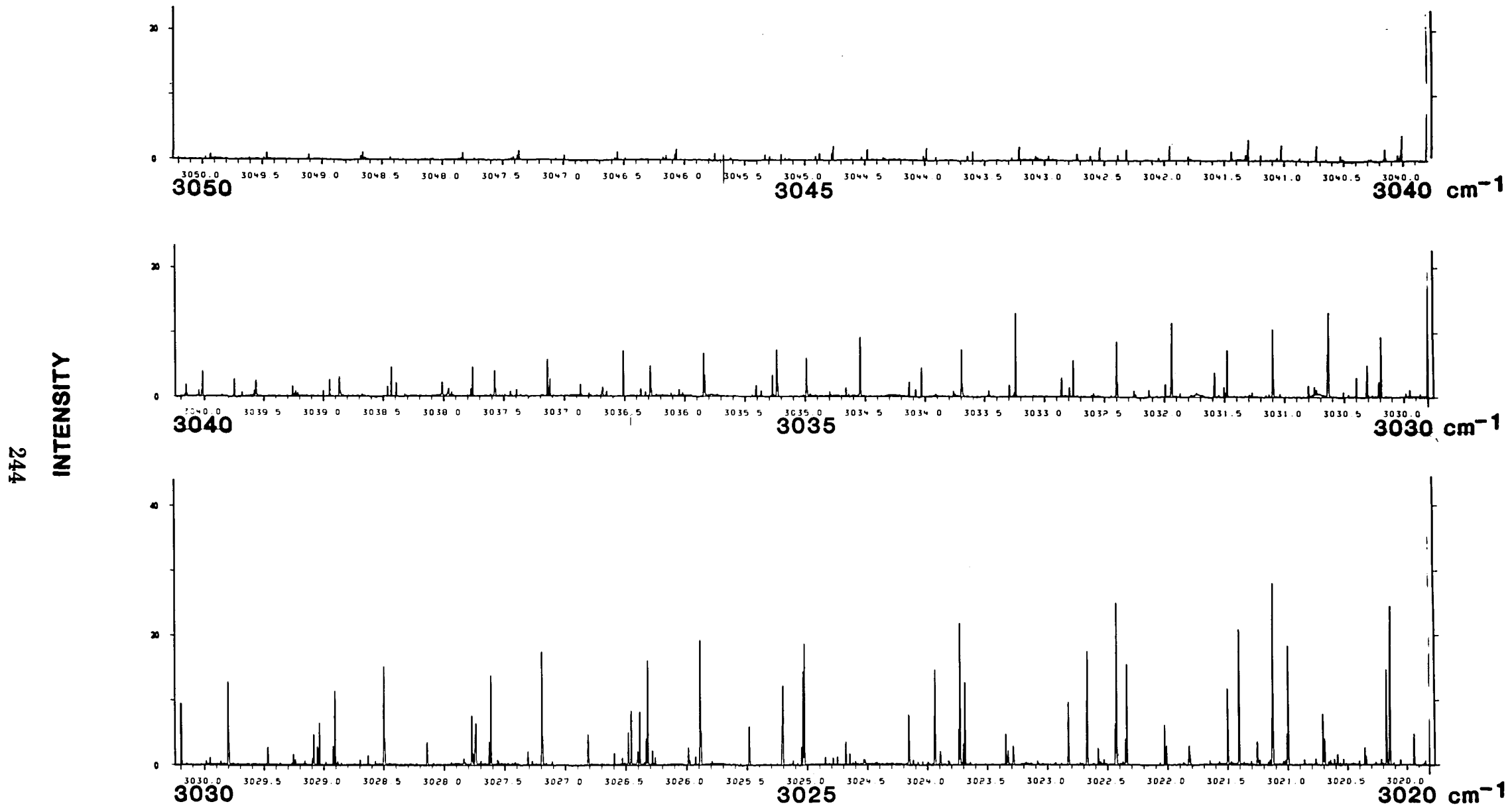

FIGURE 6. Doppler-limited spectrum of ethane at $T=119 \mathrm{~K}$. The intensity scale is in (Torr-m $)^{-1}$ uncorrected for temperature or isotopes (see text). 


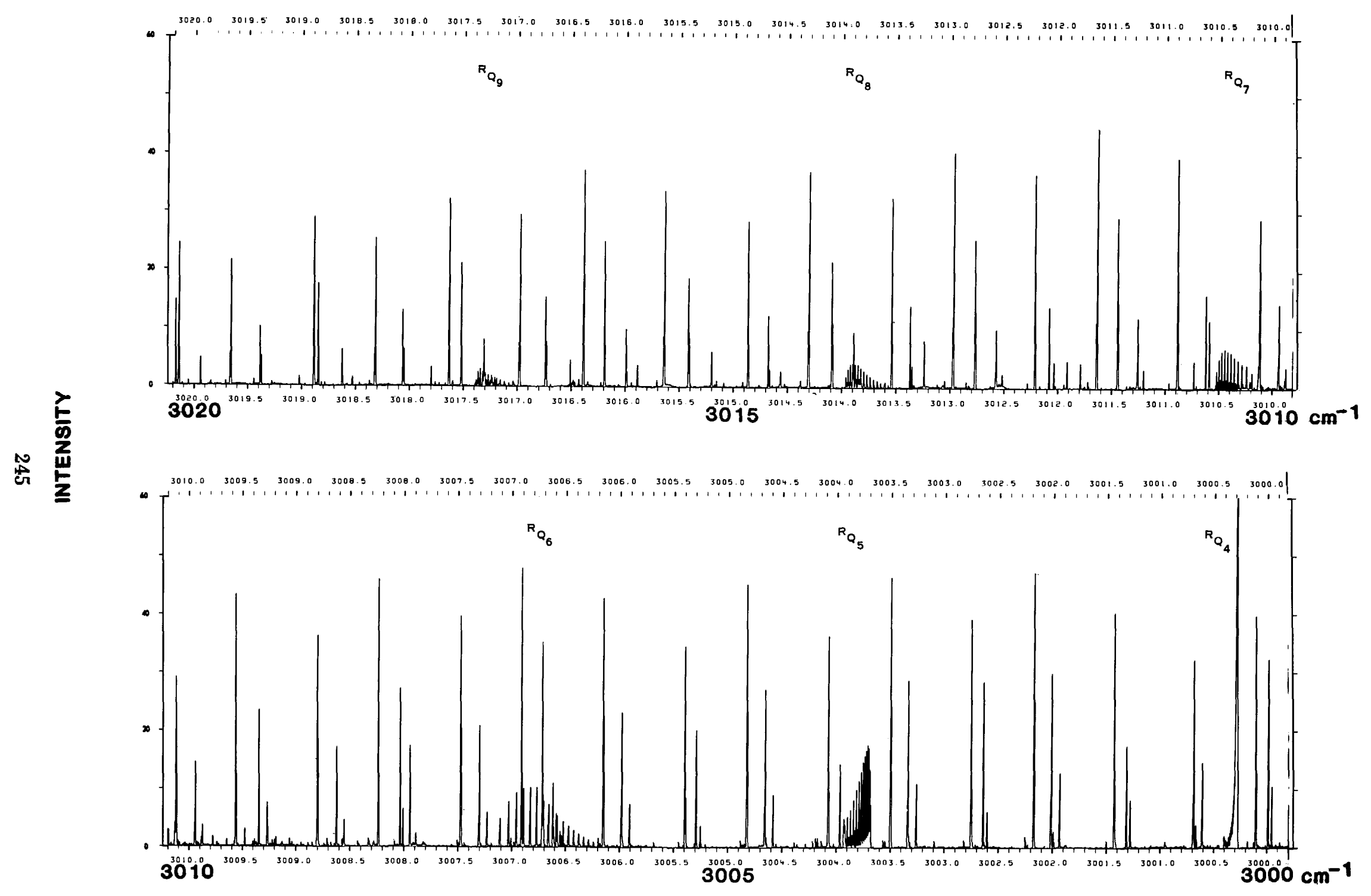

Figure 6. Continued 


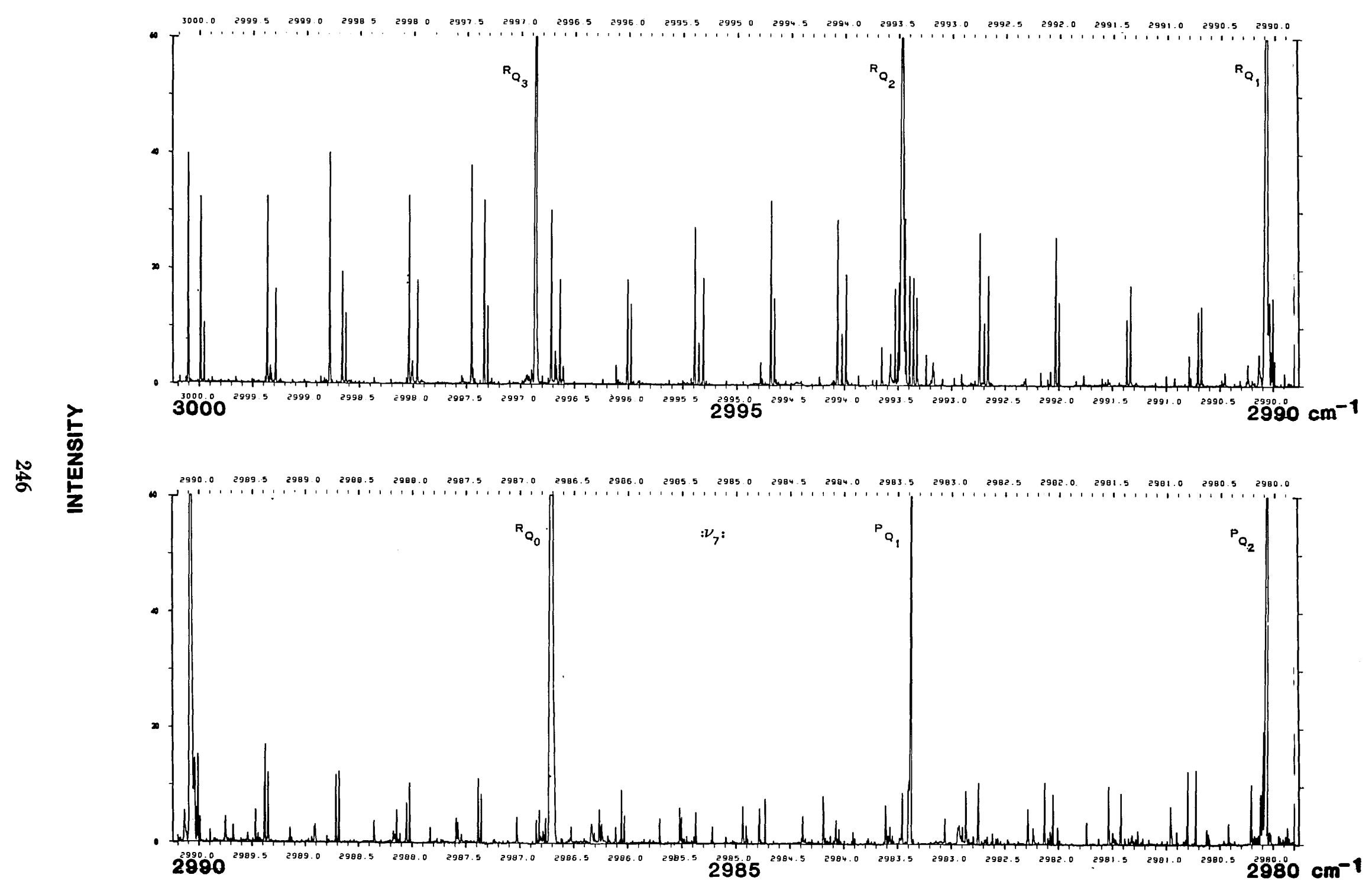

Figure 6. Continued 


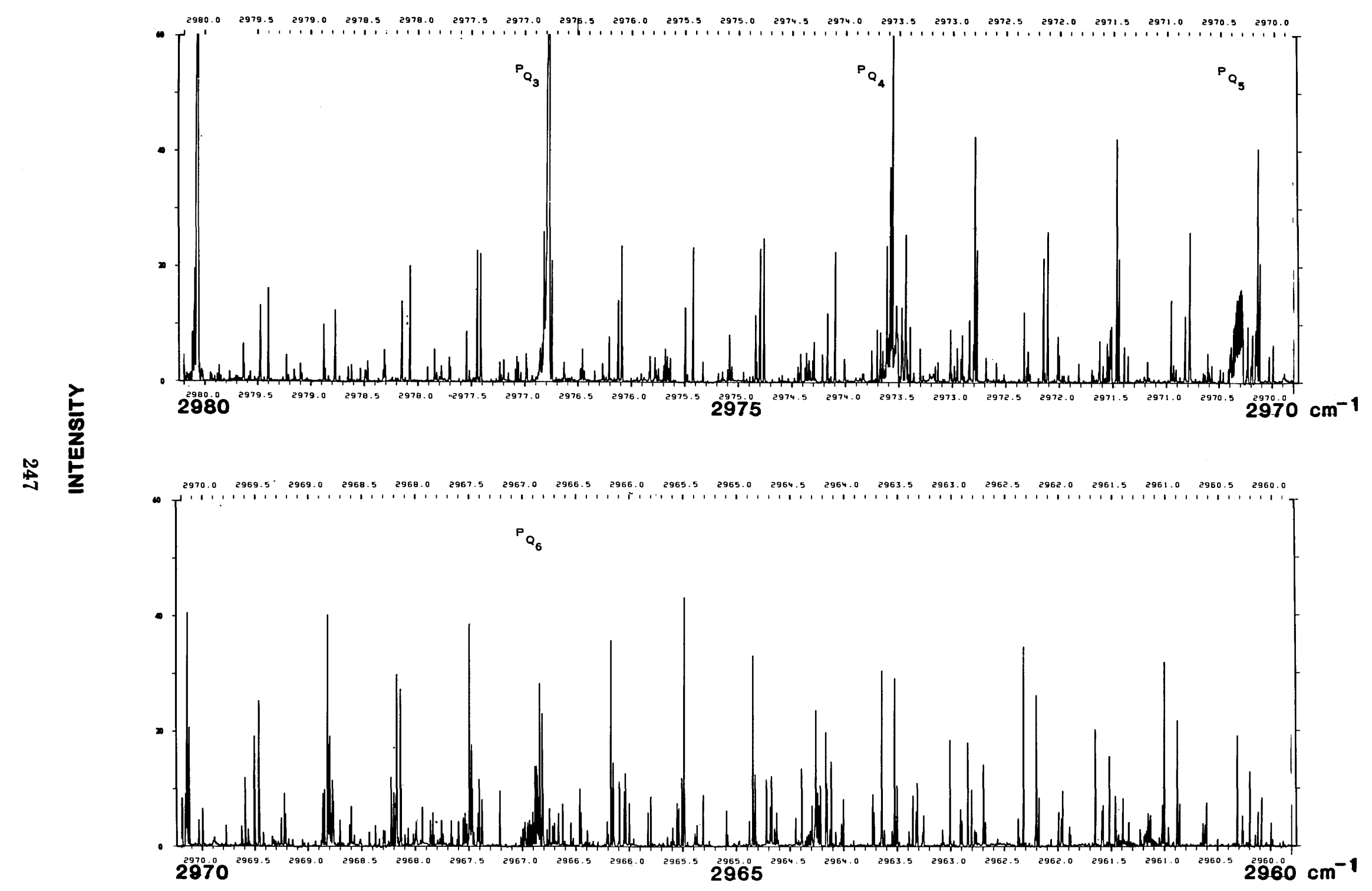

Figure 6. Continued 

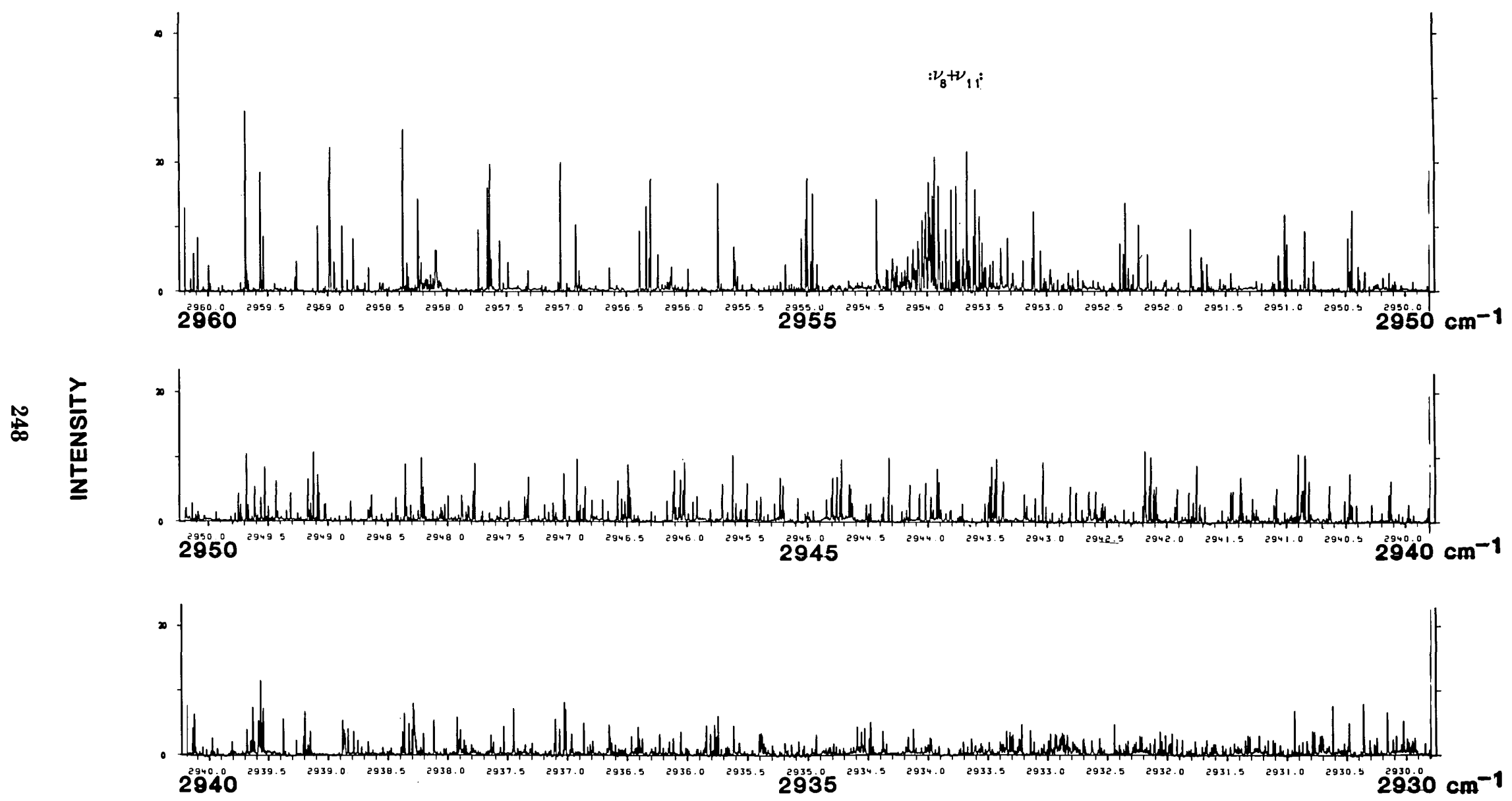


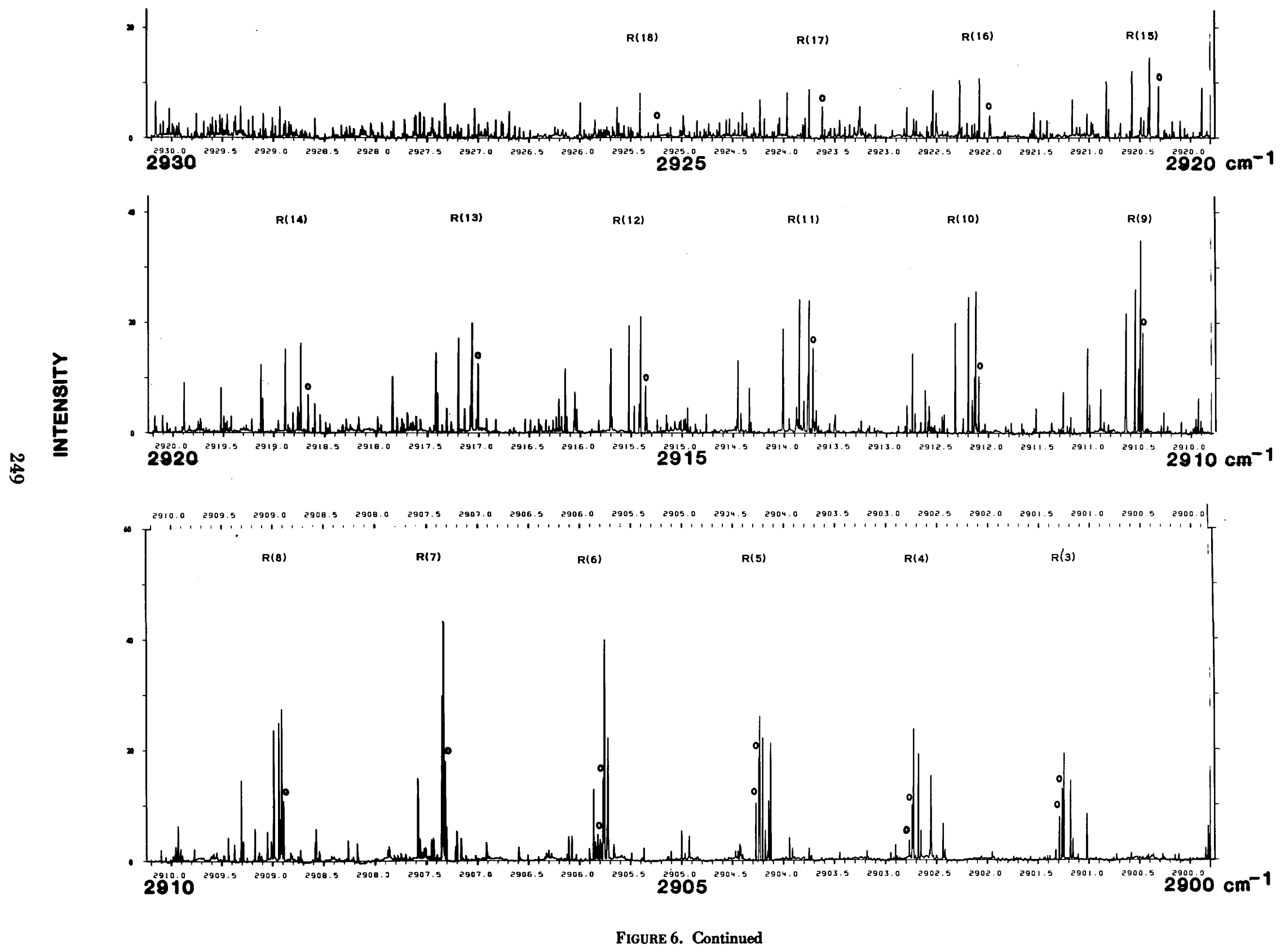



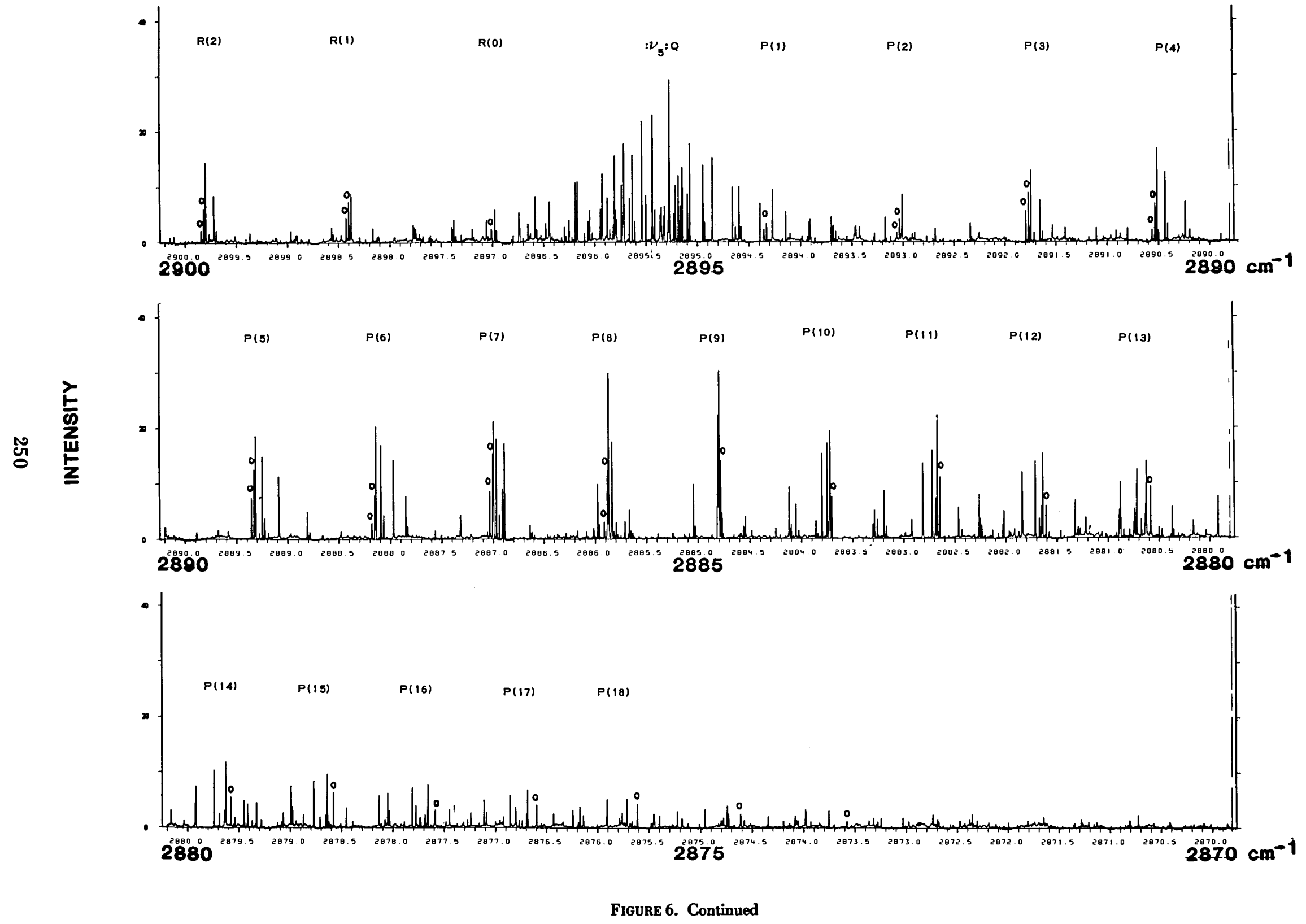
TAble 2.

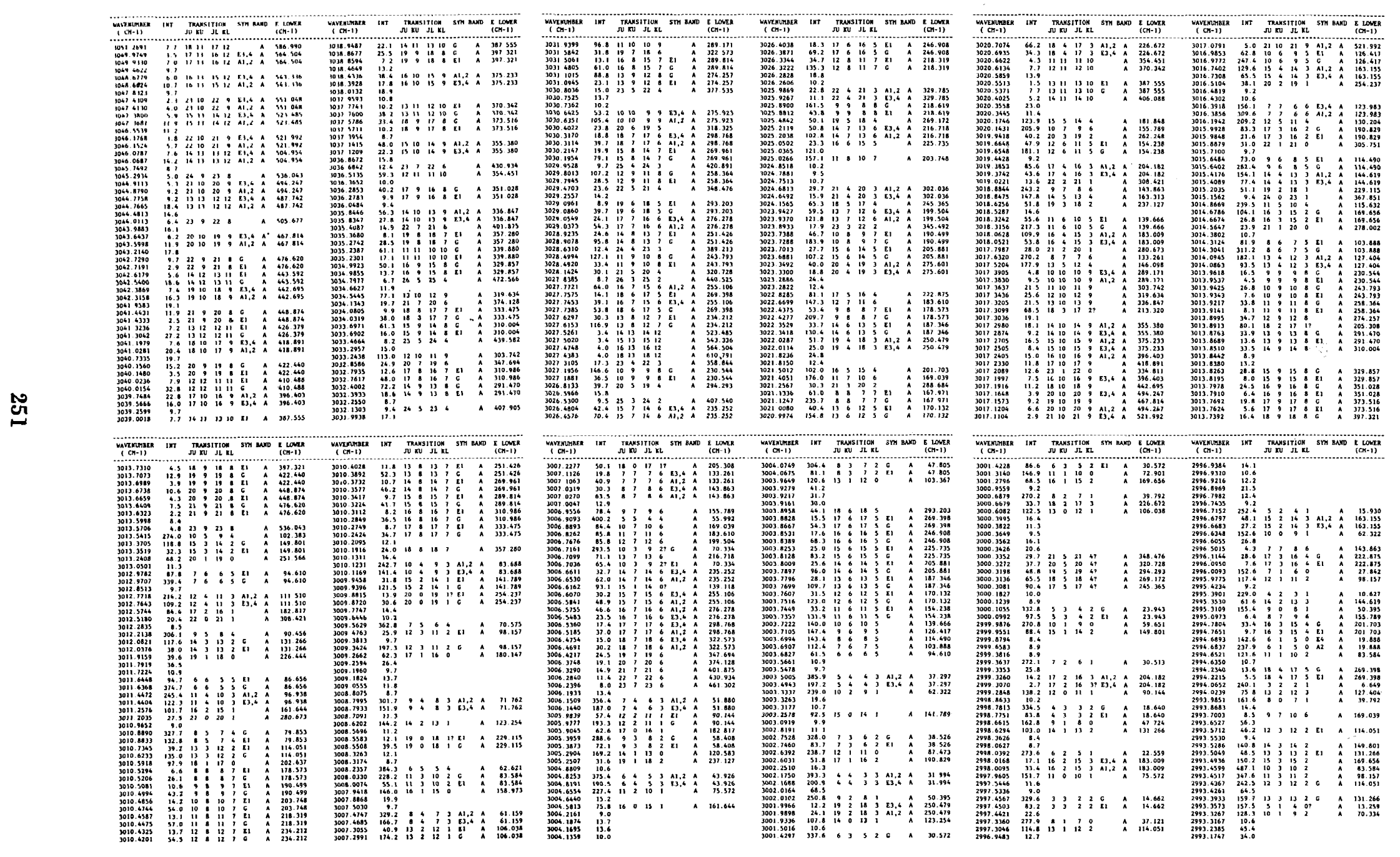



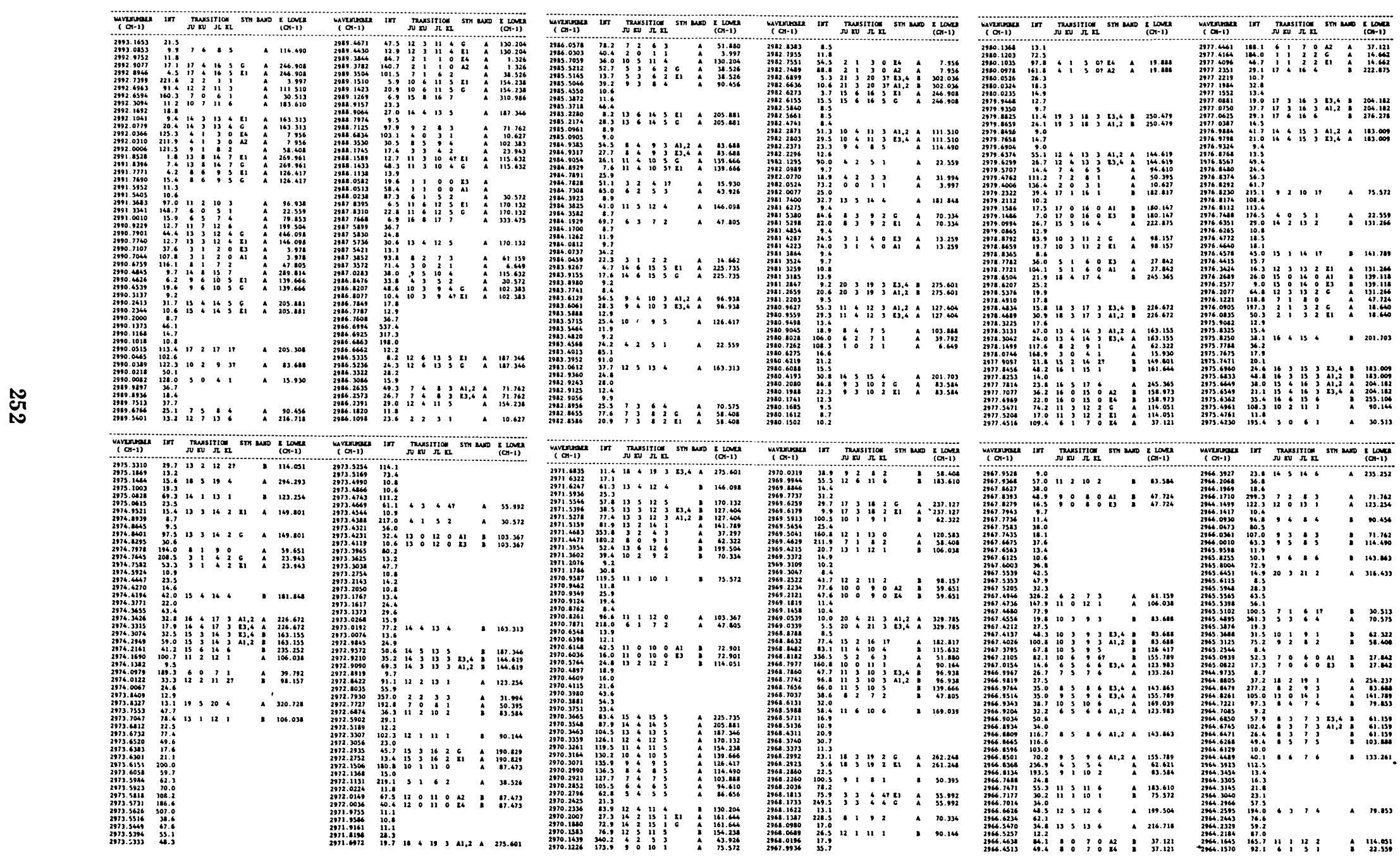


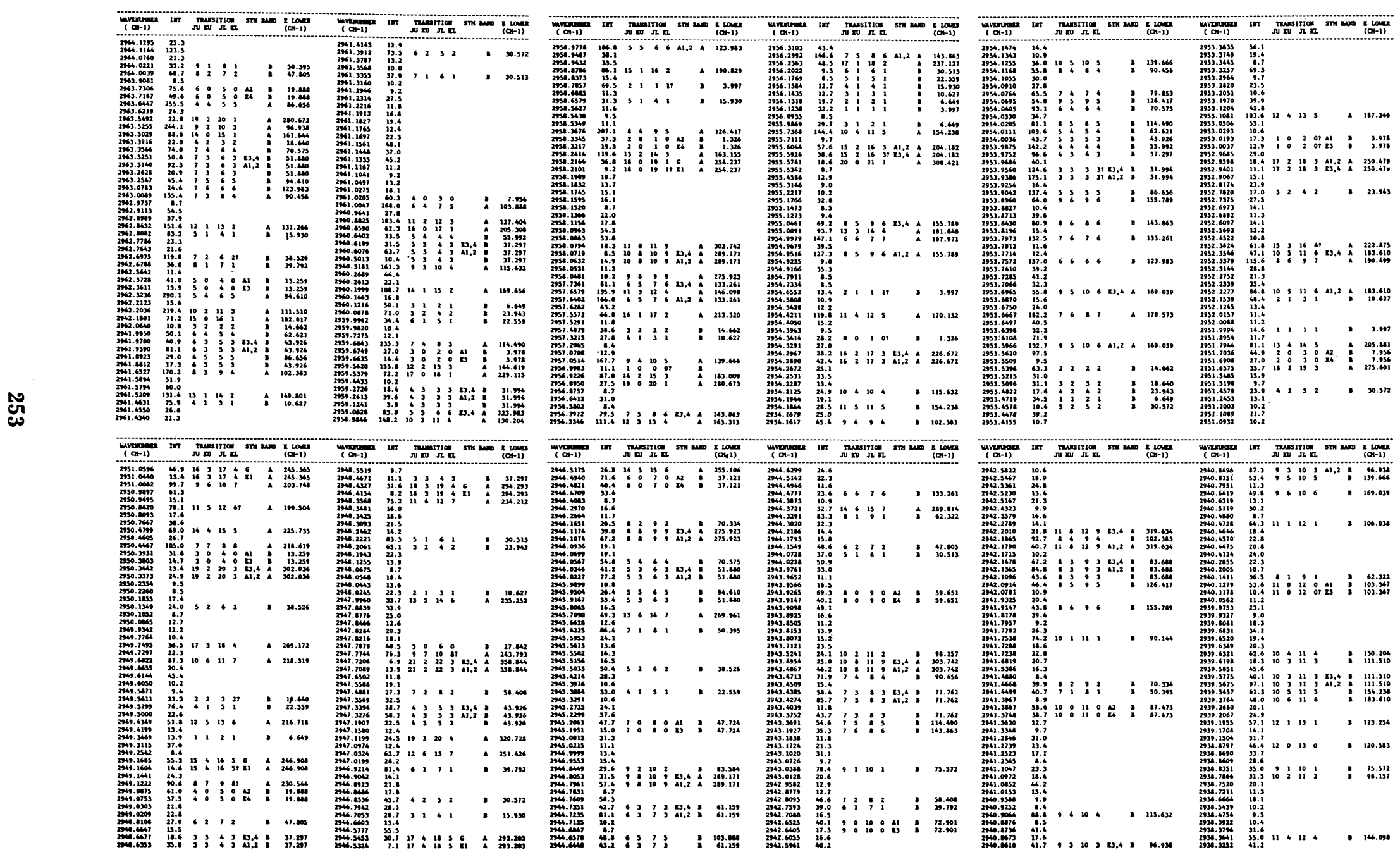


TABLE 2. continued

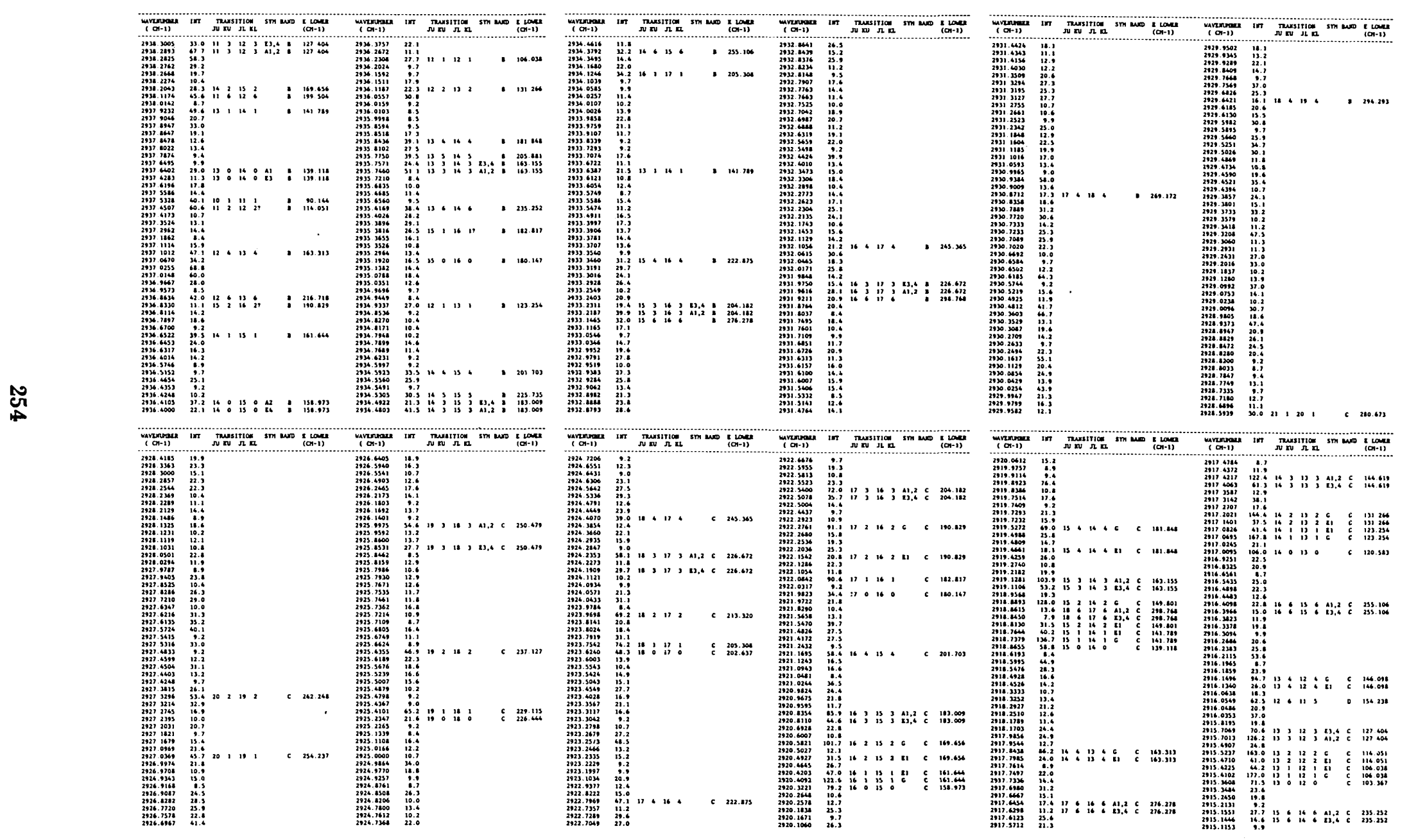




\section{TABLE 2. continued}

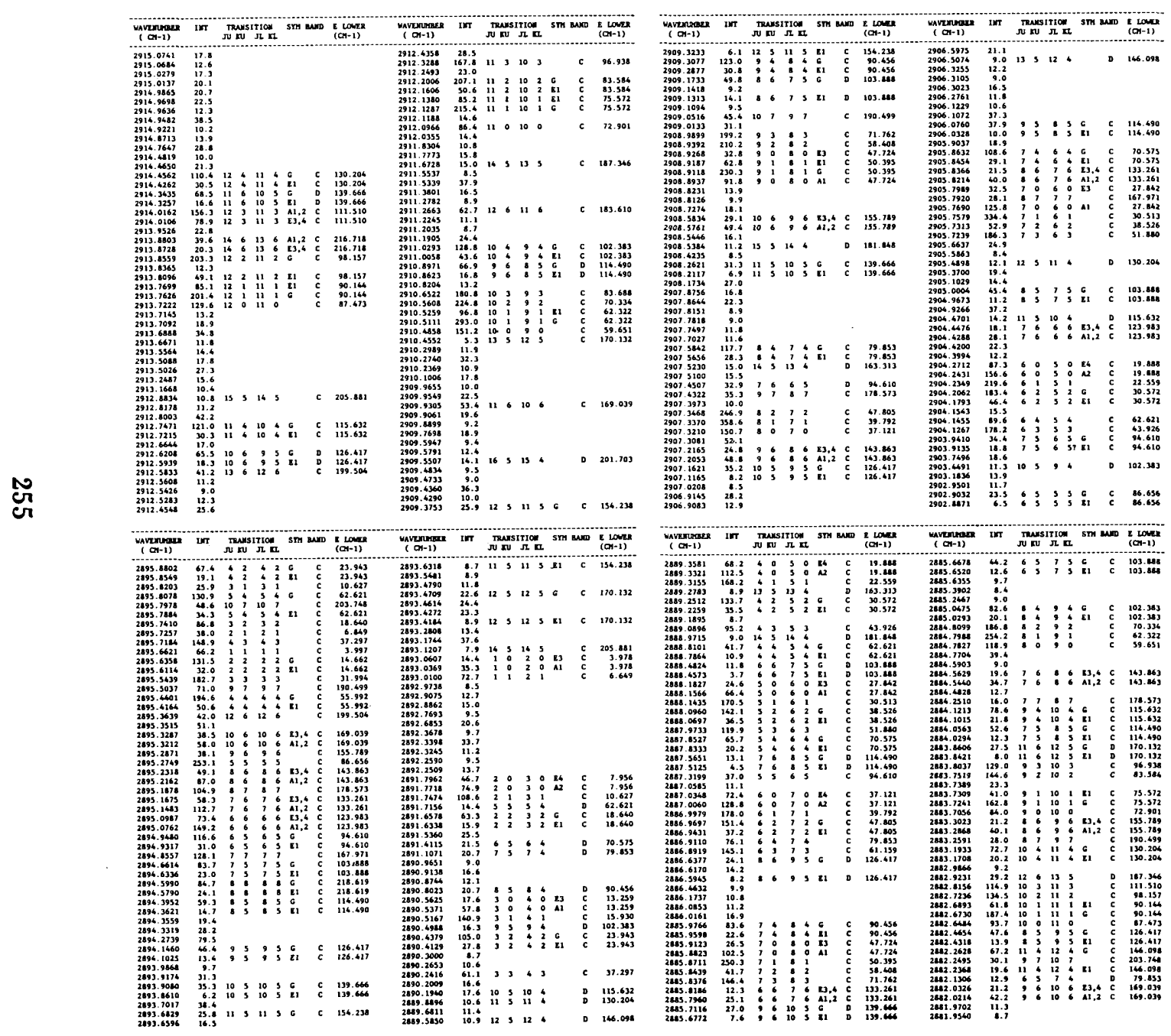

\begin{tabular}{|c|c|}
\hline \\
\hline 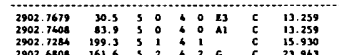 & 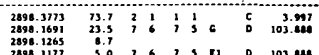 \\
\hline & 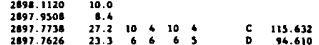 \\
\hline & 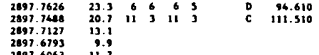 \\
\hline 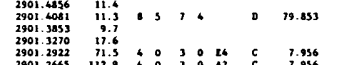 & 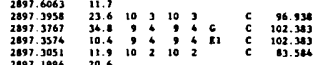 \\
\hline 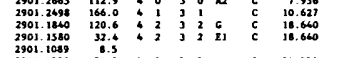 & 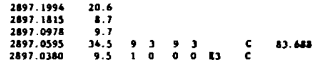 \\
\hline $\begin{array}{ll}\cdots & c \\
\cdots & 0\end{array}$ & 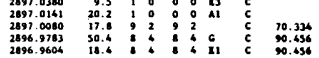 \\
\hline 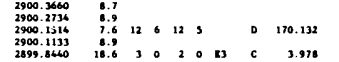 & 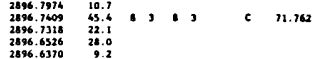 \\
\hline 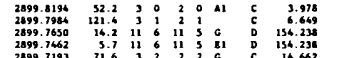 & 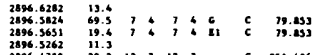 \\
\hline 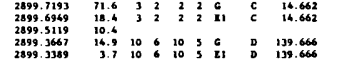 & 品? 3, 3 \\
\hline 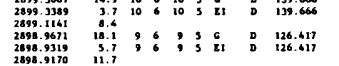 & 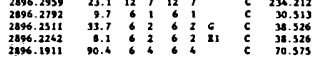 \\
\hline 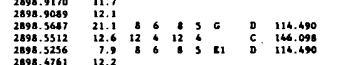 & 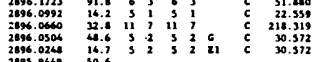 \\
\hline 42 & $3, \quad c 0.926$ \\
\hline 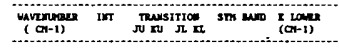 & 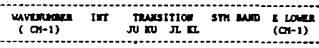 \\
\hline & 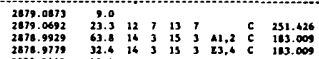 \\
\hline 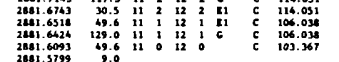 & \\
\hline $\begin{array}{llll} & \\
0\end{array}$ & \\
\hline & 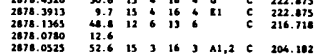 \\
\hline 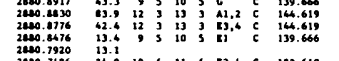 & 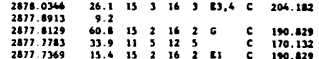 \\
\hline 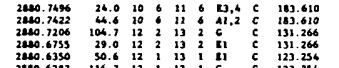 & $\begin{array}{llll} & \end{array}$ \\
\hline$i$ & 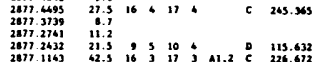 \\
\hline 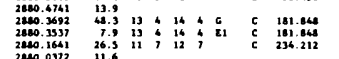 & \\
\hline 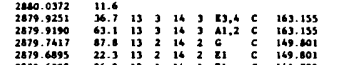 & \\
\hline 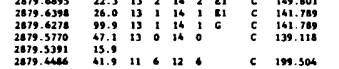 & \\
\hline 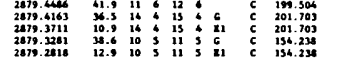 & 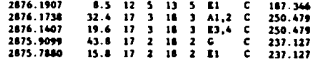 \\
\hline
\end{tabular}




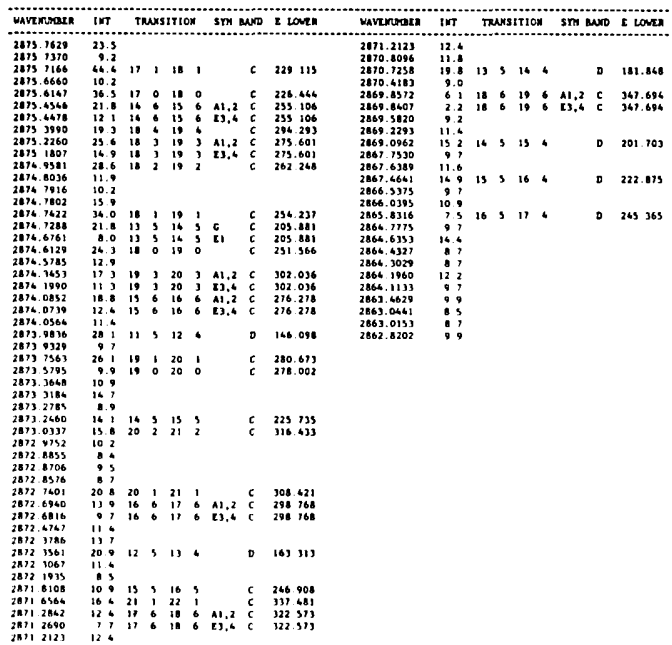

\section{References}

[1] Cole, A. R. H.; Lafferty, W. J.; Thibault, R. J. J. Mol. Spectrosc. 29, 365-374 (1969).

[2] Cole, A. R. H.; Cross, K.J.; Cugley, J. A.; Heise, H. M. J. Mol Spectrosc. 83, 233-244 (1980).

[3] Herzberg, G. Infrared and Raman Spectra of Polyatomic Molecules, D. Van Nostrand Co., New York, 1959, Chapter IV and $\mathrm{V}$.

[4] Pitzer, K. S. Dis. Faraday Soc. 10, 66-73 (1951).

[5] Pitzer, R. M. Accounts of Chem Res. in press.

[6] Weiss, S.; Leroi, G. E. J. Chem. Phys. 48, 962-967 (1967).

[7] Hirota, E.; Saito, S.; Endo, Y. J. Chem. Phys.

[8] Susskind, J. J. Mol. Spectrosc. 49, 1-17 (1974).

[9] Wilson, E. B. J. Chem. Phys. 6, 740-745 (1938).

[10] Patterson, C. W.; Flicker, H.; McDowell, R. S.; Nereson, N. Mol. Phys.

[11] Lafferty, W. J.; Hougen, J. T.; Valentin, A.; Henry, L.; MalathyDevi, V.; Das, P. P.; Rao, K. N. 36th Symposium on Molecular Spectroscopy, Paper WE2, Columbus, Ohio, June 1981.

[12] Daunt, S. J.; Jennings, D. E.; Brault, J. W.; Susskind, J.; Reuter,

D.; Blass, W. E. 36th Symposium on Molecular Spectroscopy, Paper WE3, Columbus, Ohio, June 1981.

[13] Westcott, M. R. Alberta Research Council and Hinkley, E. D. Jet Propulsion Laboratory, private communication.

[14] Toth, R. A. Jet Propulsion Laboratory, private communication.

[15] Tokunaga, A. T.; Knacke, R. F.; Ridgway, S. T.; Wallace, L. Astrophysical J. 222, 603-615 (1979).

[16] Pine, A. S. J. Opt. Soc. Amer. 64, 1683-1690 (1974).

[17] Pine, A. S. J. Opt. Soc. Amer. 66, 97-108 (1976).

[18] Pine A. S. in Laser Spectroscopy III, (J. L. Hall and J. T. Carlsten, Eds.), pp. 376-381, Springer-Verlag, New York, 1977.

[19] Coulombe, M. J.; Pine, A. S. Appl. Opt. 18, 1505-1512 (1979).

[20] Tarrago, G.; Dang-Nhu, M.; Poussigue, G.; Guelachvili, G.; Amiot, C. J. Mol. Spectrosc. 57, 246-263 (1975).

[21] Hougen, J. T. Can. J. Phys. 42, 1920-1937 (1964).

[22] Nakagawa, I.; Shimanouchi, T. J. Mol. Spectrosc. 39, 255-274 (1971).

[23] Hougen, J. T. J. Chem. Phys. 38, 1167-1173 (1963).

[24] Olson, W. B., private communication.

[25] Hougen, J. T. J. Mol. Spectrosc. 82, 92-116 (1980).

[26] Lepard, D. W.; Shaw, D. E.; Welsh, H. L. Can J. Phys. 44, 23532362 (1966). 